\title{
DUALITY IN NOETHERIAN RINGS
}

\author{
J. P. JANS ${ }^{1}$
}

0 . Introduction. Let $A$ be a module over a ring $R$. The group $A^{*}=\operatorname{Hom}_{R}(A, R)$ becomes an $R$-module (of the opposite hand from $A$ ) which we shall call the dual of $A$. If we do it again to get $A^{* *}$, there is a natural homomorphism of $A$ into $A^{* *}$,

$$
\stackrel{\stackrel{\sigma}{\rightarrow}}{\rightarrow} A^{* *}
$$

caused by considering the elements of $A$ as homomorphisms of $A^{*}$ into $R$. Following the terminology of Bass [1] we shall say that $A$ is torsionless if $\sigma$ is a monomorphism, reflexive if $\sigma$ is an isomorphism. In his paper, Bass proved the following theorem connecting duals and torsionless modules.

Shifting Theorem. Let $R$ be both right and left Noetherian, $A$ a finitely generated $R$-module, $B$ a finitely generated torsionless $R$-module, and $C^{*}$ the dual of a finitely generated $R$-module. If any one of the modules $A, B$, or $C^{*}$ is given then the other two exist and are connected by the following exact sequences

$$
\begin{aligned}
& 0 \rightarrow C^{*} \rightarrow F \rightarrow B \rightarrow 0, \\
& 0 \rightarrow B \rightarrow F^{\prime} \rightarrow A \rightarrow 0
\end{aligned}
$$

where $F$ and $F^{\prime}$ are free.

Since we shall make such heavy use of this theorem and the techniques used in its proof, we shall now make the standing assumptions that every ring we consider will be both right and left Noetherian and that every module will be finitely generated. The notation and terminology will follow that of Cartan and Eilenberg [2] although we shall usually drop the ring from Hom and Ext since we shall not change rings.

In this paper, we shall be concerned with several questions arising out of duality theory. In $\$ 1$ we shall consider various conditions which insure that certain modules are reflexive. A useful tool is the exact sequence $0 \rightarrow A \rightarrow A^{* *} \rightarrow \operatorname{Ext}^{1}(B, R) \rightarrow 0$ which holds for torsion-

Received by the editors November 8, 1960.

1 This research was supported by the United States Air Force through the Air Force Office of Scientific Research of the Air Research and Development Command, under Contract No. AF 49(638)-921. Reproduction in whole or in part is permitted for any purpose of the United States Government. 
less $A$ and a suitable choice of $B$ (Theorem 1.1). Thus it becomes clear that reflexiveness is related to the vanishing of $\operatorname{Ext}^{1}(B, R)$ (Corollary 1.3) or of its dual (Corollary 1.5). Also in this section we show that the third dual $A^{* * *}$ is the direct sum of the first dual and the dual of a module of the form $\operatorname{Ext}^{1}(B, R)$.

Since it came up rather naturally in the first section to consider $\operatorname{Ext}^{1}(B, R)$ as a module (of the opposite hand to $B$ ), in the second section we study modules of this form. In answer to the question of which modules are of this form, we get the best results about modules which are farthest from being reflexive. We show that if $A^{*}=0$ then there exists $B$ such that $A=\operatorname{Ext}^{1}(B, R)$ and moreover, if $A \neq 0, B$ can be chosen to have projective dimension one. As a corollary to this we can describe rings with the property $\operatorname{lf} P D(R)=0$ (see [1] for definition) by the condition that for each right module $A$, if $A \neq 0$ then $A^{*} \neq 0$.

We conclude the section by considering a variation of Nunke's theorem [5].

1. Which modules are reflexive? In this section we shall consider various conditions which imply that certain classes of modules are reflexive. The following theorem states that for torsionless $A, A^{* *} / A$ $=\operatorname{Ext}^{1}(B, R)$ for a suitable $B$. To emphasize the dual roles of $A$ and $B$ we state the theorem in the following form:

THEOREM 1.1. If $A$ is a torsionless module, there exists $B$, torsionless of the opposite hand from $A$, such that the sequences

$$
\begin{aligned}
& 0 \rightarrow A \stackrel{\sigma}{\rightarrow} A^{* *} \rightarrow \operatorname{Ext}^{1}(B, R) \rightarrow 0 \\
& 0 \rightarrow B \stackrel{\sigma}{\rightarrow} B^{* *} \rightarrow \operatorname{Ext}^{1}(A, R) \rightarrow 0
\end{aligned}
$$

are exact.

Proof. Resolve the module $A$ by the sequence $0 \rightarrow M \rightarrow i F \rightarrow A \rightarrow 0$ where $F$ is free. Since $A$ is torsionless, $M$ is closed in $F$ [1]. Now form the homomorphisms into $R$ to get $0 \rightarrow A^{*} \rightarrow F^{*} \rightarrow B \rightarrow 0$ exact where $B=F^{*} / A^{*}$. We dualize again but this time we do it in two different ways:

$$
\begin{aligned}
& 0 \rightarrow B^{*} \stackrel{j}{\rightarrow} F^{* *} \rightarrow A \rightarrow 0 \\
& 0 \rightarrow B^{*} \stackrel{j}{\rightarrow} F^{* *} \rightarrow A^{* *} \rightarrow \operatorname{Ext}^{1}(B, R) \rightarrow 0 .
\end{aligned}
$$

The first of these is essentially the technique of Bass in showing that 
$M=B^{*}[1]$, the second sequence is the exact sequence of homology for Ext in the first variable. Combining these two sequences we obtain $0 \rightarrow A \rightarrow^{\sigma} A^{* *} \rightarrow \operatorname{Ext}^{1}(B, R) \rightarrow 0$ exact. Note that the injection of $A$ into $A^{* *}$ is "as homomorphisms on $A^{*}$ " so the injection is the natural one. The corresponding statement with the roles of $A$ and $B$ interchanged comes from the symmetry of the two exact sequences

$$
\begin{aligned}
& 0 \rightarrow B^{*} \rightarrow F \rightarrow A \rightarrow 0 \\
& 0 \rightarrow A^{*} \rightarrow F^{*} \rightarrow B \rightarrow 0
\end{aligned}
$$

which occurred in the above argument.

One of the reasons for stressing the symmetric form of the above theorem is that not only does $\operatorname{Ext}^{1}(B, R)$ measure the difference between $A$ and $A^{* *}$, but that every torsionless $B$ will appear for a suitable choice of $A$. Another thing which should be noted is that, although there are many possible choices for $B$, they all give rise to the same module $\operatorname{Ext}^{1}(B, R)$ which depends only on $A$.

The following are two corollaries to the theorem obtained from the application of Bass' Shifting Theorem. The first was proved by Dieudonné [3].

Corollary 1.2. $A=A^{* *}$ for all $A$ if and only if $R$ is a Quasi Frobenius ring.

Proof. If $R$ is Quasi Frobenius then $\operatorname{Ext}^{1}(B, R)=0$ for all $B$ and therefore $A=A^{* *}$ for all torsionless $A$ by Theorem 1.1. Now form the sequence $0 \rightarrow C \rightarrow F \rightarrow A \rightarrow 0$ with arbitrary $A$, and free $F$, and torsionless $C$ according to the first half of the Shifting Theorem. The vanishing of $\operatorname{Ext}^{1}(X, R)$ for all $X$ implies that both of the dual sequences

$$
0 \rightarrow A^{*} \rightarrow F^{*} \rightarrow C^{*} \rightarrow 0
$$

and

$$
0 \rightarrow C^{* *} \rightarrow F^{* *} \rightarrow A^{* *} \rightarrow 0
$$

are exact. But since we have already shown that $C=C^{* *}, F=F^{* *}$ it follows that $A=A^{* *}$. Going in the other direction, if $A=A^{* *}$ for all $A$ then by Theorem 1.1 we see that $\operatorname{Ext}^{1}(B, R)=0$ for all torsionless modules $B$. But since $B=B^{* *}$, every module is torsionless and it follows that $R$ is both left and right self injective. For the rings under consideration this is equivalent to being Quasi Frobenius [4].

If perfect duality $\left(A=A^{* *}\right.$ for all $A$ ) fails, one might wonder under what conditions the next best thing is available. That is, when are torsionless modules reflexive? The following gives an answer. 
COROLLARY 1.3. The following are equivalent:

1. $A=A^{* *}$ for every torsionless left module $A$.

2. The right injective dimension of $R$ is $\leqq 1$.

Proof. By Bass' Shifting Theorem, we see that right injective dimension of $R \leqq 1$ is equivalent to $\operatorname{Ext}^{1}(B, R)=0$ for all torsionless right modules $B$. By Theorem 1.1, this is equivalent to $A=A^{* *}$ for all torsionless left modules $A$.

The class of duals of modules is, in general, smaller than the class of torsionless modules. We are led therefore to considering conditions under which every dual is reflexive. This brings in the third dual and with it a lot more structure. For, in addition to the natural injection

$$
A^{*} \stackrel{\tau}{\rightarrow} A^{* * *}
$$

of the first dual into the third, there is also the homomorphism

$$
A^{* * *} \stackrel{\sigma^{*}}{\rightarrow} A^{*}
$$

induced by the natural injection $A \rightarrow A^{* *}$ (assuming $A$ torsionless). In the proof of the following theorem we shall show that the composition $\sigma^{*} \circ \tau$ is identity on $A^{*}$.

TheOREM 1.4. ${ }^{2}$ If $A$ is torsionless and $B$ is chosen to satisfy the conclusion of Theorem 1.1 then $A^{* * *}=A^{*} \oplus\left[\operatorname{Ext}^{1}(B, R)\right]^{*}$.

Proof. By Theorem 1.1 we have the exact sequence $0 \rightarrow A \rightarrow A^{*} * *$ $\rightarrow \operatorname{Ext}^{1}(B, R) \rightarrow 0$ which induces a sequence $0 \rightarrow\left[\operatorname{Ext}^{1}(B, R)\right]^{*} \rightarrow A^{* * *}$ $\rightarrow \sigma^{*} A^{*}$ which is also exact.

For notational convenience, we introduce at this point the inner product notation. If $x$ is in $A^{* *}$ and $y$ in $A^{* * *}$, we denote the image of $x$ under $y$ in $R$ by $(x, y)$. Then if $a$ is in $A, b$ in $A^{*}$ we see that $(\sigma(a), \tau(b))=(a, b)$ where the right hand side is the image of $a$ under $b$. Consider now the mapping $\sigma^{*} \circ \tau$; let $a$ be in $A, b$ in $A^{*}$ and form $\left(a, \sigma^{*} \circ \tau(b)\right)$. By the definition of $\sigma^{*}$ this equals $(\sigma(a), \tau(b))$ which in turn is $(a, b)$ by the above remark. Hence we can conclude that $\sigma^{*} \circ \tau$ is the identity on $A^{*}$. Thus the sequence

$$
0 \rightarrow\left[\operatorname{Ext}^{1}(B, R)\right]^{*} \rightarrow A^{* * *} \stackrel{\sigma^{*}}{\rightarrow} A^{*} \rightarrow 0
$$

is not only exact, but it also splits. This provides the conclusion of the theorem.

${ }^{2}$ S. Chase informs me that the splitting part of Theorem 1.4 is in Bass' dissertation at the University of Chicago. 
As a corollary to the theorem, we can establish precisely when duals are reflexive.

COROLlaRy 1.5. The following are equivalent:

1. Every dual of a left module is reflexive.

2. $\left[\operatorname{Ext}^{1}(B, R)\right]^{*}=0$ for all torsionless right modules $B$.

3. $\left[\operatorname{Ext}^{2}(C, R)\right] *=0$ for all right modules $C$.

Proof. The equivalence of 2 and 3 comes from the Shifting Theorem. The equivalence of 1 and 2 follows from Theorem 1.4.

2. Which modules are Exts? The next question one might ask is what modules $A$ are of the form $\operatorname{Ext}^{1}(B, R)$ for a suitable $B$ of the opposite hand from $A$. The following theorem gives one property of a module which is an Ext.

Theorem 2.1. If $\operatorname{dim} A=n$ for $1 \leqq n<\infty$ then $\left[\operatorname{Ext}^{n}(A, R)\right]^{*}=0$.

Proof. The proof is by induction on $n$. For $n=1$ we can resolve $A$ with a pair of projectives

$$
0 \rightarrow P \stackrel{j}{\rightarrow} P_{0} \rightarrow A \rightarrow 0 \text {, exact. }
$$

This induces the exact sequence of homology

$$
0 \rightarrow A^{*} \rightarrow P_{0}^{*} \stackrel{j^{*}}{\rightarrow} P^{*} \rightarrow \operatorname{Ext}^{1}(A, R) \rightarrow 0 .
$$

We now star the tail end of this sequence to obtain

$$
0 \rightarrow\left[\operatorname{Ext}^{1}(A, R)\right]^{*} \rightarrow P_{0} \stackrel{j}{\rightarrow} P
$$

which is also exact. In the above sequence we have substituted $P=P^{* *}, j=j^{* *}$ and $P_{0}=P_{0}^{* *}$ since the $P^{\prime}$ s are projective and therefore reflexive. But since $\operatorname{Ker} j=0$ we see that $\left[\operatorname{Ext}^{1}(A, R)\right]^{*}=0$.

Assume now that the theorem has been established for integers less than $n$ and let the dimension of $A$ be $n \geqq 2$. We shift back by the exact sequence $0 \rightarrow M \rightarrow P \rightarrow A \rightarrow 0$ where $P$ is projective and $M$ has dimension $n-1$. By induction, we may assume that $\left[\mathrm{Ext}^{n-1}(M, R)\right]^{*}$ $=0$. However, the exact sequence of homology in the first variable of Ext shows that $\operatorname{Ext}^{n}(A, R)$ is isomorphic to $\operatorname{Ext}^{n-1}(M, R)$.

We can now establish a partial answer to the question of which modules are Ext of something.

Theorem 2.2. If $A^{*}=0$ then there exists $B$ such that $A=\operatorname{Ext}^{1}(B, R)$ (and, incidentally, $B$ can be chosen of dimension one if $A \neq 0$ ). 
Proof. If $A=0$ then taking $B$ projective will do the job. If $A \neq 0$ then $A$ is not projective and we can start to resolve $A$ with a pair of free modules

$$
F \stackrel{\delta}{\rightarrow} F_{0} \rightarrow A \rightarrow 0 .
$$

This induces the exact sequence

$$
0 \rightarrow A^{*} \rightarrow F_{0}{ }^{*} \stackrel{\delta^{*}}{\rightarrow} F^{*} \rightarrow F^{*} / \operatorname{Im} \delta^{*} \rightarrow 0 .
$$

By the assumption that $A^{*}=0$ the above sequence can be shortened to $0 \rightarrow F_{0}^{*} \rightarrow^{\delta^{*}} F^{*} \rightarrow B \rightarrow 0$ where we take for $B$ the module $F^{*} / \operatorname{Im} \delta^{*}$. Now apply the stars all over again to obtain

$$
0 \rightarrow B^{*} \rightarrow F^{* *} \stackrel{\delta^{* *}}{\longrightarrow} F_{0}^{* *} \rightarrow \operatorname{Ext}^{1}(B, R) \rightarrow 0 .
$$

But we note that the $F^{\prime}$ s are free hence reflexive and $\delta^{* *}=\delta$, hence we can conclude that $\operatorname{Ext}^{1}(B, R)=A$.

We can put Theorems 2.1 and 2.2 together to obtain a new characterization of rings of finitistic dimension zero. Following Bass, we shall define $\operatorname{lf} P D(R)=\operatorname{Sup} \operatorname{dim} A$ where the $A$ 's range over the left $R$ modules which are finite dimensional and finitely generated.

CoROllary 2.3. IfPD $(R)=0$ if and only if for each right module $A \neq 0, A^{*} \neq 0$.

Proof. If $R$ has a left module $A$ of dimension $n$ for $1 \leqq n<\infty$ then Theorem 2.1 implies that $\left[\operatorname{Ext}^{n}(A, R)\right]^{*}=0$. Also we know in this case that $\operatorname{Ext}^{n}(A, R) \neq 0$ by [2, p. 123]. Conversely, if $R$ has a right module $A \neq 0$ for which $A^{*}=0$ then by the proof of Theorem 2.2 there exists a left module $B$ of dimension 1 such that $\operatorname{Ext}^{1}(B, R)=A$. This completes the proof of the corollary.

We would like to mention here that the problem of which modules are of the form $\operatorname{Ext}^{n}(A, R)$ is closely related to some excellent work done by Nunke [5]. In that paper he was concerned which modules over a Dedekind ring are of the form $\operatorname{Ext}(A, C)$ for modules $A$ and $C$. His class of rings is more restrictive than ours but he does not put chain conditions on the modules he considers.

In his paper Nunke also proved the following concerning Dedekind rings which are not complete discrete valuation rings: If $A^{*}=0$ $=\operatorname{Ext}^{1}(A, R)$ then $A=0$. No finiteness conditions are made concerning the module $A$. We shall say that a ring $R$ satisfies Nunke's condition if $A^{*}=0=\operatorname{Ext}^{r}(A, R)$ for $r>1$ implies that $A=0$ for $R$-modules $A$. Similarly we shall say that $R$ satisfies Nunke's condition for some 
special class of $R$-modules if the above statement is true for all $R$ modules in the class.

It is not hard to show that the rings we are considering here all have Nunke's condition on the class of finitely generated modules of finite projective dimension. See $[2$, p. 123]. The proof of Theorem 2.2 indicates that we can say a little more in the case that the duals of one dimensional modules are finite dimensional.

THEOREM 2.4. If $R$ has the property that duals of one dimensional modules are finite dimension then $R$ satisfies Nunke's condition for finitely generated modules.

Proof. It is enough, from the remark above, to show that if $A^{*}=0$ then $A$ has finite projective dimension. But in the proof of Theorem 2.2 we encountered the sequence $0 \rightarrow B^{*} \rightarrow F \rightarrow F_{0} \rightarrow A \rightarrow 0$ where $B$ has dimension 1 . The hypothesis about duals then implies that $A$ has finite dimension.

\section{REFERENCES}

1. H. Bass, Finitistic dimension and a homological generalization of semi-primary rings, Trans. Amer. Math. Soc. vol. 95 (1960) pp. 466-488.

2. H. Cartan and S. Eilenberg, Homological algebra, Princeton University Press, 1956.

3. J. Dieudonné, Remarks on quasi-Frobenius rings, Illinois J. Math. vol. 2 (1958) pp. 346-354.

4. S. Eilenberg and T. Nakayama, On the dimension of modules and algebras. II, Frobenius algebras and quasi-Frobenius rings, Nagoya Math. J. vol. 9 (1955) pp. 1-16.

5. R. J. Nunke, Modules of extensions over Dedekind rings, Illinois J. Math. vol. 3 (1959) pp. 222-241.

Princeton University and

UNIVERSITY OF WASHINGTON 